\title{
Commento Editoriale
}

\author{
Vincenzo M. Mastronardi \\ Psichiatra, Psicoterapeuta, Ipnologo Clinico, Docente di Psichiatria Corso di laurea inf. Canale H Università di Roma \\ Sapienza, Italy
}

Il presente 2020 non è stato soltanto un anno di pandemia limitato alla rarefazione dei rapporti umani, finanziari e di fertili ascese esistenziali, bensì ha rappresentato un vero e proprio momento di congelamento intrapsichico ed interpersonale in grado di bloccare emozionalmente ogni processo di fisiologico consolidamento delle migliori evoluzioni sociali e di miglioramento di se stessi in ogni suo intento ottimistico di costruzione basilare secondo le abituali coordinate della stessa evoluzione antropologica. Difatti ne ha risentito anche il processo di motivazione culturale. Si sono potuti registrare infatti palesi deflessioni finanche nelle iscrizioni a più corsi di aggiornamento e di perfezionamento. Gli uffici chiusi e con lavoro in Smart Working non sempre vicarianti la funzionalità richiesta e la stasi esistenziale conseguente con contagio comportamentale strisciante, ha rappresentato un duro colpo alle giuste competizioni interpersonali in grado di motivarsi vicendevolmente. Per fortuna il desiderio di canalizzare le proprie ricerche già in corso, hanno rappresentato una fertile occasione per i nostri allievi, i quali in questo numero della nostra Rivista di Psicopatologia forense, Medicina Legale e Criminologia hanno fornito il loro contributo scientifico spesso oggetto delle loro apprezzabili tesi. Pur se ragioni contingenti legate all'inerzia dei collegamenti tra più uffici amministrativi hanno rappresentato un vero problema, per fortuna il digitale e il
Web sia pure con i propri limiti sono riusciti nel miglior modo possibile a compensare ogni limitazione di sorta. È per questa ragione che per quest'anno nonostante tutto, siamo lieti di fornire ai nostri lettori i lavori del 2020 sia pure in formato digitale, ma non per questo meno organizzato proprio grazie alla tenacia della Casa Editrice. La presente prestigiosa rivista ricordiamo al lettore che vede i suoi natali nella sua primissima veste redazionale fin dal lontano 1974 per i tipi di Minerva Medica e grazie al prof. Franco Granone dell'Università di Torino dal quale chi scrive la ereditò già dal 1996.

Vincenzo M. Mastronardi Psichiatra, Criminologo Clinico, già Direttore della Cattedra di Psicopatologia forense,

Università di Roma Sapienza

Docente incaricato di "Psichiatria" Corso di Laurea H inf.

Università di Roma Sapienza

Docente di Criminologia Clinica Scuola di Specializzazione in medicina Legale, Università di Roma Sapienza Docente del Corso di Laurea Magistrale in Investigazione, Criminalità e Sicurezza Internazionale, Università degli Studi Internazionali di Roma
Correspondence: Vincenzo M. Mastronardi.

E-mail: vincenzo.mastronardi@gmail.com

Received for publication: 27 November 2020.

Accepted for publication: 27 November 2020.

This article is distributed under the terms of the Creative Commons Attribution Noncommercial License (by-nc 4.0) which permits any noncommercial use, distribution, and reproduction in any medium, provided the original author(s) and source are credited.

${ }^{\circ}$ Copyright: the Author(s), 2020

Licensee PAGEPress, Italy

Rivista di Psicopatologia Forense, Medicina Legale, Criminologia $2020 ; 25: 556$

doi:10.4081/psyco.2020.556 